\title{
Deformation Behavior of the Alloys under Simple and Combined Loading Conditions at Various Deformation Rate
}

\author{
Takeshi Iwamoto ${ }^{1, *(D)}$ and Tao Suo ${ }^{2,3, *}$ \\ 1 Academy of Science and Technology, Hiroshima University, 1-4-1 Kagamiyama, Higashi-Hiroshima, \\ Hiroshima 739-8527, Japan \\ 2 School of Aeronautics, Northwestern Polytechnical University, Xi'an 710072, China \\ 3 National Joint International Reasearch Laboratory of Impact Dynamics and Its Engineering Applications, \\ Xi'an 710072, China \\ * Correspondence: iwamoto@mec.hiroshima-u.ac.jp (T.I.); suotao@nwpu.edu.cn (T.S.); Tel.: +81-82-424-7576 \\ (T.I.); +86-159-0920-8902 (T.S.)
}

Received: 14 September 2018; Accepted: 17 September 2018; Published: 18 September 2018

\section{Introduction and Scope}

Clarifying the mechanical behavior of alloys is quite important to enhance their performance and thus expand their application in various fields. Actually, alloys undergo not only simple, but also quite complicated mechanical loading conditions via combinations of simple loading modes with proportional and/or nonproportional histories. Studies on a penetration/ballistic problem are frequently found for this kind of categories because the metals used there deform at higher strain rates and the problem basically arises due to its complicated loading conditions/histories. Additionally, products with high performances are quite useful to any situations involving not only low-speed, but also high-speed deformation. For example, their applications make it possible to avoid fatal accidents involving transportation equipment moving rapidly. Furthermore, the high-speed deformation will be utilized for an increase in efficiency of their production. In their cases, strain rate sensitivity in the inelastic deformation of alloys holds the key, and it is important to clarify dynamic or impact effects of the phenomena under complicated deformation processes imposed. In the present Special Issue, research works related to the deformation behavior of alloys with higher deformation rates are invited, as well as interdisciplinary works concerning these topics. Fundamental research works on testing methods and computational simulations are also included.

This Special Issue is composed of articles reporting on new and progressive research results of particular classes of fundamental and complicated deformation behaviors of alloys at various deformation rates and their applications.

\section{Contributions}

In total, eight articles [1-8] have been presented in the present Special Issue. Their target metals are broad, ranging from an advanced steel [1] to alloys composed of light metals such as magnesium [2], titanium [3], and aluminum [3-6], as well as alloys of a smart Ni-Ti system with a shape memory effect [7] and depleted uranium [8]. Almost all target metals are commonly used in industry. The strain rate in their works is imposed to these alloys mainly in a higher range from dynamic to impact levels, such as 10 to $10^{3} \mathrm{~s}^{-1}$. This means it is easier to apply the target metals to the higher speed deformation. On the other hand, the finite element computation, experimental studies, or their combinations are performed in the articles. Similar to the target metals themselves, the approaches used to study them are also broad. 
The following four experimental works $[1,2,4,8]$ discuss from metallurgical points of view. The microstructural studies are done as well by using TEM, Electron Back Scatter Diffraction (EBSD), or Energy Dispersive Spectroscopy (EDS). Wang et al. [1] studies the strain rate effect on the tensile behaviors of a high-specific-strength steel (HSSS) with dual-phase microstructure. Major tensile properties show the positive sensitivity with respect to the strain rate ranging from $10^{-4}$ to $10 \mathrm{~s}^{-1}$. They found that hardening by means of back stress plays an important role for this HSSS due to load transfer and strain partitioning between harder and softer phases. Rao et al. [2] examined AZX312 magnesium alloys with different initial processing in terms of its compressive strength and hot working behavior. They developed maps for the processing at the various temperatures and at strain rates ranging from $10^{-4}$ to $10 \mathrm{~s}^{-1}$. As a result, the compressive strength is significantly improved by a disintegrated melt deposition processing. At higher strain rates, there is an advantage to obtain the additional workability in designing forming processes which facilitate faster production. The effect of strain rate on the mechanical properties of AA5xxx series aluminum alloys was studied experimentally in a wide strain rate range of $10^{-4}$ to $10^{3} \mathrm{~s}^{-1}$ at room temperature [4]. The AA5xxx alloys display a negative strain rate sensitivity of flow stress and serration derived from the Portevin-Le Chatelier (PLC) effect. By using the uniaxial hardening model for the negative strain rate sensitivity, the authors found that the flow stress may change in the dynamic strain rate range. Adiabatic shear bands (ASBs) in projectiles made of a U-0.75 wt \% Ti alloy were studied [8]. The sample was launched to penetrate steel targets, and the distribution of ASBs in collected samples was studied. The authors found that the "self-sharpening effect" in the projectiles is caused by the distribution of the ASBs, which becomes a softer zone compared with the matrix as a result of a microhardness test.

On the other hand, the following studies [3,5-7] are combined works involving both experimental and simulation approaches, sometimes with modelling of a hardening model. In some works, a fractographic study and dimensional analysis are introduced. Wang et al. [3] proposed a new method to determine the parameters in the revised Johnson-Cook failure model for 2618 aluminum and Ti-6Al-4V titanium alloys. In this method, both the simulation of designed specimens with different notches using the finite element model and the digital image correlation method with the micro speckle were employed. They conducted the quasi-static and dynamic experiments under different temperatures and the simulations for the ballistic impact tests to validate the accuracy of the parameters. The influence of the stress state on the deformation and failure behaviors of three aluminum alloys was investigated [5]. In this study, tension-torsion tests were conducted in a wide range of stress triaxiality as well as the Lode parameter. Impact experiments were further conducted with different geometries of projectiles and the experiments were simulated by using finite element models to evaluate the predictability of three failure criteria. As a fundamental research work on a testing method, Gao and Iwamoto [6] showed the possibility to modify the Taylor impact test for measuring the stress-strain curve of pure aluminum by only single number of the test through the finite element simulation. In this study, a bilinear distribution of internal force in the specimen was assumed and they introduced measurements of impact force by a Hopkinson pressure bar and deformation profile by a high-speed camera to solve the problems related to nonuniform deformation and necessity of numerous numbers of tests. The shock effects in laser shock processing of a Ni-Ti shape memory alloy were studied through both dimensional analysis and finite element simulation [7]. The relationship between the plastically affected depth and peak pressure, one of the dimensionless parameters, was validated with experimental results conducted in this study. The numerical results clarifies the scaling law of shock effects on dimensionless parameters quantitatively.

\section{Concluding Remarks and Outlook}

As described in the introduction, the penetration/ballistic problems are appropriately and commonly used to deal with the strain rate sensitivity of metals, as well as complicated loading conditions at the same time. The inclusion of this kind of works is appreciated and they must be continued for complete understanding. In addition, the anomalies of deformation behavior derived 
from its strain rate sensitivity, such as the PLC effect and ASBs, is quite interesting and still unclarified. In this Issue, new methods are proposed to understand the deformation or failure behavior of the alloys. Not only the penetration problems, but also the other types of nonuniform deformation will be included in testing methods. The introduction of nonuniform deformation will be developed more and more, and further new methods will be established by using both simulation and experimental approaches because of the difficulty of the problems. Moreover, utilization of high-speed deformation in the thermomechanical processing will be extended not only to reduce the time, but also to extract new performance or abilities of the alloys. From the above insights, we would conclude that new and progressive research works were collected successfully, and are hereby collated in this Special Issue.

Finally, as guest editors, we sincerely would like to thank all the authors for their valuable contributions to the present Special Issue.

Acknowledgments: Special thanks to the editorial team in Metals, especially Sherry Liu and Will Li, for their valuable assistance and support during the preparation of this Special Issue.

Conflicts of Interest: The authors declare no conflict of interest.

\section{References}

1. Wang, W.; Ma, Y.; Yang, M.; Jiang, P.; Yuan, F.; Wu, X. Strain rate effect on tensile behavior for a high specific strength steel: From quasi-static to intermediate strain rates. Metals 2018, 8, 11. [CrossRef]

2. Rao, K.P.; Suresh, K.; Prasad, Y.V.R.K.; Hort, N.; Gupta, M. Enhancement of strength and hot workability of AZX312 magnesium alloy by disintegrated melt deposition (DMD) processing in contrast to permanent mold casting. Metals 2018, 8, 437. [CrossRef]

3. Wang, C.; Suo, T.; Li, Y.; Xue, P.; Tang, Z. A new experimental and numerical framework for determining of revised J-C failure parameters. Metals 2018, 8, 396. [CrossRef]

4. Yamada, H.; Kami, T.; Mori, R.; Kudo, T.; Okada, M. Strain rate dependence of material strength in AA5xxx series aluminum alloys and evaluation of their constitutive equation. Metals 2018, 8, 576. [CrossRef]

5. Rodriguez-Millan, M.; Garcia-Gonzalez, D.; Rusinek, A.; Arias, A. Influence of stress state on the mechanical impact and deformation behaviors of aluminum alloys. Metals 2018, 8, 520. [CrossRef]

6. Gao, C.; Iwamoto, T. Finite element analysis on a newly-modified method for the Taylor impact test to measure the stress-strain curve by the only single test using pure aluminum. Metals 2018, 8, 642. [CrossRef]

7. Wang, X.; Xia, W.; Wu, X.; Huang, C. Scaling law in laser-induced shock effects of NiTi shape memory alloy. Metals 2018, 8, 174. [CrossRef]

8. Wang, B.; Dong, Y.; Huang, G. An investigation on the adiabatic shear bands in depleted U-0.75 wt \% Ti alloy under dynamic loading. Metals 2018, 8, 145. [CrossRef]

(C) 2018 by the authors. Licensee MDPI, Basel, Switzerland. This article is an open access article distributed under the terms and conditions of the Creative Commons Attribution (CC BY) license (http:/ / creativecommons.org/licenses/by/4.0/). 